\title{
The Convex $(\delta, L)$ Weak Contraction Mapping Theorem and its Non-Self Counterpart in Graphic Language
}

\section{Clement Boateng Ampadu}

31 Carrolton Road, Boston, MA 02132-6303, USA; e-mail: drampadu@ @otmail.com

\begin{abstract}
Let $(X, d)$ be a metric space. A map $T: X \mapsto X$ is said to be a $(\delta, L)$ weak contraction [1] if there exists $\delta \in(0,1)$ and $L \geq 0$ such that the following inequality holds for all $x, y \in X$ :

$$
d(T x, T y) \leq \delta d(x, y)+L d(y, T x) .
$$

On the other hand, the idea of convex contractions appeared in [2] and [3]. In the first part of this paper, motivated by [1]-[3], we introduce a concept of convex $(\delta, L)$ weak contraction, and obtain a fixed point theorem associated with this mapping. In the second part of this paper, we consider the map is a non-self map, and obtain a best proximity point theorem. Finally, we leave the reader with some open problems.
\end{abstract}

\section{Introduction and Preliminaries}

The higher-order fixed point theory [4] is inspired by [5]. In particular, the idea of higher-order Banach mapping was defined as follows:

Definition 1.1. [5] Let $(X, d)$ be a metric space. A map $T: X \mapsto X$ is called an rth-order Banach mapping if for all $x, y \in X, 0 \leq c_{q}<1,0 \leq q \leq r-1$, and $r \in \mathbb{N}$, the following inequality holds

Received: February 5, 2019; Accepted: February 25, 2019; Published online: February 26, 2019 2010 Mathematics Subject Classification: 47H10.

Keywords and phrases: $(\delta, L)$ weak contraction, convex contraction, non-self map, graph, fixed point theorem, best proximity point theorem.

Copyright (C) 2019 Clement Boateng Ampadu. This is an open access article distributed under the Creative Commons Attribution License, which permits unrestricted use, distribution, and reproduction in any medium, provided the original work is properly cited. 


$$
d\left(T^{r} x, T^{r} y\right) \leq \sum_{q=0}^{r-1} c_{q} d\left(T^{q} x, T^{q} y\right)
$$

with $\sum_{q=0}^{r-1} c_{q}<1$.

Remark 1.2. A map $T: X \mapsto X$ is called a convex contraction [2]-[3], if $r=2$ in the definition immediately above.

By these observations we introduce the following

Definition 1.3. Let $(X, d)$ be a metric space. A map $T: X \mapsto X$ is called an rth$\operatorname{order}(\delta, L)$ weak contraction mapping if for all $x, y \in X, 0<\delta_{q}<1, L_{q} \geq 0$, $0 \leq q \leq r-1$, and $r \in \mathbb{N}$, the following inequality holds

$$
d\left(T^{r} x, T^{r} y\right) \leq \sum_{q=0}^{r-1}\left\{\delta_{q} d\left(T^{q} x, T^{q} y\right)+L_{q} d\left(T^{q} y, T^{q+1} x\right)\right\}
$$

with $\sum_{q=0}^{r-1} \delta_{q}<1$

Remark 1.4. If $r=2$ in the definition immediately above, then we say $T: X \mapsto X$ is a convex $(\delta, L)$ weak contraction mapping. Note that if $r=1$ in the above definition, then $T: X \mapsto X$ is a $(\delta, L)$ weak contraction [1].

Also we recall the following results associated with the $(\delta, L)$ weak contraction

Theorem 1.5. [1] Let $(X, d)$ be a complete metric space and $T: X \mapsto X$ be an almost contraction, that is, a mapping for which there exist a constant $\delta \in[0,1)$ and some $L \geq 0$ such that for all $x, y \in X$

$$
d(T x, T y) \leq \delta d(x, y)+L d(y, T x) .
$$

Then

(a) $\operatorname{Fix}(T)=\{x \in X: T x=x\} \neq \varnothing$.

(b) For any $x_{0} \in X$, the Picard iteration $\left\{x_{n}\right\}_{n=0}^{\infty}$ given by $x_{n+1}=T x_{n}, n=1,2, \ldots$ converges to some $x^{*} \in \operatorname{Fix}(T)$. 
(c) The following estimate holds

$$
d\left(x_{n+i-1}, x^{*}\right) \leq \frac{\delta^{i}}{1-\delta} d\left(x_{n-1}, x_{n}\right)
$$

$n=0,1,2, \ldots ; i=1,2, \ldots$

Theorem 1.6. [6] Let $(X, d)$ be a complete metric space and $T: X \mapsto X$ be a weak contraction for which there exist a constant $\theta \in(0,1)$ and some $L_{1} \geq 0$ such that for all $x, y \in X$

$$
d(T x, T y) \leq \theta d(x, y)+L_{1} d(x, T x) .
$$

Then

(a) Thas a unique fixed point, that is, $F(T)=\left\{x^{*}\right\}$.

(b) For any $x_{0} \in X$, the Picard iteration $\left\{x_{n}\right\}_{n=0}^{\infty}$ given by $x_{n+1}=T x_{n}, n=1,2, \ldots$ converges to $x^{*}$.

(c) The a priori and a posteriori error estimates holds

$$
d\left(x_{n}, x^{*}\right) \leq \frac{\delta^{n}}{1-\delta} d\left(x_{0}, x_{1}\right)
$$

for $n=0,1,2, \ldots$

$$
d\left(x_{n}, x^{*}\right) \leq \frac{\delta}{1-\delta} d\left(x_{n-1}, x_{n}\right)
$$

for $n=1,2, \ldots$

(d) The rate of convergence of the Picard iteration is given by

$$
d\left(x_{n}, x^{*}\right) \leq \theta d\left(x_{n-1}, x^{*}\right)
$$

for $n=1,2, \ldots$

Now let $W$ and $V$ be two nonempty subsets of a metric space $(X, d)$ and let $S: W \mapsto V$ be a non-self map. If $W \cap V$ is nonempty, then the equation $S x=x$ may not have a solution. Naturally the following arises

Question 1.7. How far is the distance between $x$ and $S x$ ? 
The problem of global optimization for determining the minimum value of the distance $d(x, S x)=\min \{d(x, y): x \in W$ and $y \in V\}$ is the study of best proximity point theory. Since the early paper of [7], many best proximity point theorems have been obtained, and for example see references [9-23] contained in [8].

Notation 1.8. Throughout this paper

(a) $W$ and $V$ will denote nonempty subsets of a metric space $(X, d)$.

(b) $d(W, V):=\inf \{d(x, y): x \in W$ and $y \in V\}$.

(c) $W_{0}=\{x \in W: d(x, y)=d(W, V)$ for some $y \in V\}$.

(d) $V_{0}=\{y \in V: d(x, y)=d(W, V)$ for some $x \in W\}$.

The notion of proximal contraction appeared in [9], now we introduce the following

Definition 1.9. Let $S: W \mapsto V$ be a non-self mapping. We say $S$ is a proximal convex $(\delta, L)$ weak contraction if there exists $\delta_{0}, \delta_{1} \in(0,1), L_{0}, L_{1} \geq 0$, and $u_{1}, u_{2}, x, y \in W$ such that $d\left(u_{1}, S x\right)=d(W, V)$ and $d\left(u_{2}, S y\right)=d(W, V)$ implies

$$
d\left(S u_{1}, S u_{2}\right) \leq \delta_{0} d(x, y)+L_{0} d\left(y, u_{1}\right)+\delta_{1} d(S x, S y)+L_{1} d\left(S y, S u_{1}\right) .
$$

The notion of $G$-proximal Kannan mapping appeared in [8], now we introduce the following

Definition 1.10. Let $(X, d)$ be a metric space, and $G=(V(G), E(G))$ be a directed graph such that $V(G)=X$. A non-self mapping $S: W \mapsto V$ is called a $G$-proximal convex $(\delta, L)$ weak contraction, if there exists $\delta_{0}, \delta_{1} \in(0,1)$ and $L_{0}, L_{1} \geq 0$, such that $(x, y) \in E(G), d(u, S x)=d(W, V)$ and $d(v, S y)=d(W, V)$ implies

$$
d(S u, S v) \leq \delta_{0} d(x, y)+L_{0} d(y, u)+\delta_{1} d(S x, S y)+L_{1} d(S y, S u),
$$

where $x, y, u, v \in W$.

Definition 1.11. [8] Let $(X, d)$ be a metric space and $G=(V(G), E(G))$ be a directed graph such that $V(G)=X$. A non-self mapping $S: W \mapsto V$ is called proximally G-edge-preserving, if for each $x, y, u, v \in W,(x, y) \in E(G), d(u, S x)=$ $d(W, V)$ and $d(v, S y)=d(W, V)$ implies $(u, v) \in E(G)$. 
The rest of this paper is organized as follows. In the next section we obtain a fixed point theorem associated with the convex $(\delta, L)$ weak contraction, and a best proximity point theorem for its non-self version endowed with a graph. We close this paper with some open problems suggested in Section 3.

\section{Main Result}

\subsection{A fixed point theorem}

Theorem 2.1. Let $(X, d)$ be a metric space, and $T: X \mapsto X$ be a convex $(\delta, L)$ weak contraction mapping, that is, $T$ satisfies

$$
d\left(T^{2} x, T^{2} y\right) \leq \delta_{0} d(x, y)+L_{0} d(y, T x)+\delta_{1} d(T x, T y)+L_{1} d\left(T y, T^{2} x\right)
$$

for all $x, y \in X$ with $0<\delta_{0}, \delta_{1}<1, L_{0}, L_{1} \geq 0$, and $\delta_{0}+\delta_{1}<1$. If $(X, d)$ is complete, then the fixed point of $T$ exists. If, in addition, $T$ is a convex $(\delta, L)$ weak contraction such that there exists $0<\delta_{0}, \delta_{1}<1, L_{0}^{*}, L_{1}^{*} \geq 0$, with $\delta_{0}+\delta_{1}<1$ satisfying

$$
d\left(T^{2} x, T^{2} y\right) \leq \delta_{0} d(x, y)+L_{0}^{*} d(x, T x)+\delta_{1} d(T x, T y)+L_{1}^{*} d\left(T x, T^{2} x\right),
$$

then the fixed point is unique.

Proof. Define $x_{n+1}=T x_{n}=T^{2} x_{n-1}$ for all $n \in \mathbb{N}$, and observe we have the following

$$
\begin{aligned}
d\left(x_{n+1}, x_{n+2}\right)= & d\left(T^{2} x_{n-1}, T^{2} x_{n}\right) \\
\leq & \delta_{0} d\left(x_{n-1}, x_{n}\right)+L_{0} d\left(x_{n}, T x_{n-1}\right)+\delta_{1} d\left(T x_{n-1}, T x_{n}\right) \\
& +L_{1} d\left(T x_{n}, T^{2} x_{n-1}\right) \\
= & \delta_{0} d\left(x_{n-1}, x_{n}\right)+L_{0} d\left(x_{n}, x_{n}\right)+\delta_{1} d\left(x_{n}, x_{n+1}\right)+L_{1} d\left(x_{n+1}, x_{n+1}\right) \\
= & \delta_{0} d\left(x_{n-1}, x_{n}\right)+\delta_{1} d\left(x_{n}, x_{n+1}\right) \\
\leq & \left(\delta_{0}+\delta_{1}\right) \max \left\{d\left(x_{n-1}, x_{n}\right), d\left(x_{n}, x_{n+1}\right)\right\} \\
= & \left(\delta_{0}+\delta_{1}\right) d\left(x_{n}, x_{n+1}\right) .
\end{aligned}
$$


Set $h:=\left(\delta_{0}+\delta_{1}\right)$, and observe by induction we have $d\left(x_{n}, x_{n+1}\right) \leq h^{n} d\left(x_{0}, x_{1}\right)$ for all $n \in \mathbb{N}$. For $n, m \in \mathbb{N}$ with $n<m$ we deduce the following

$$
\begin{aligned}
d\left(x_{n}, x_{m}\right) & \leq d\left(x_{n}, x_{n+1}\right)+\cdots+d\left(x_{m-1}, x_{m}\right) \\
& \leq h^{n} d\left(x_{0}, x_{1}\right)+\cdots+h^{m-1} d\left(x_{0}, x_{1}\right) \\
& \leq\left(h^{n}+h^{n+1}+\cdots\right) d\left(x_{0}, x_{1}\right) \\
& =\frac{h^{n}}{1-h} d\left(x_{0}, x_{1}\right) .
\end{aligned}
$$

Since $h<1$, if we take limits in the above inequality as $n, m \rightarrow \infty$ we deduce that $\left\{x_{n}\right\}$ is Cauchy, and since $X$ is complete, there is $v \in X$ such that $\lim _{n \rightarrow \infty} x_{n}=v$. Now we show the fixed point exist. Suppose $v$ is a fixed point of $T$ but not of $T^{2}$, then we know $d(v, T v)=0$, but $d\left(v, T^{2} v\right)>0$. Now observe we have the following

$$
\begin{aligned}
0 & <d\left(v, T^{2} v\right) \\
& \leq d\left(v, x_{n+1}\right)+d\left(x_{n+1}, T^{2} v\right) \\
& =d\left(v, x_{n+1}\right)+d\left(T^{2} x_{n-1}, T^{2} v\right) \\
& \leq d\left(v, x_{n+1}\right)+\delta_{0} d\left(x_{n-1}, v\right)+L_{0} d\left(v, T x_{n-1}\right)+\delta_{1} d\left(T x_{n-1}, T v\right)+L_{1} d\left(T v, T^{2} x_{n-1}\right) \\
& =d\left(v, x_{n+1}\right)+\delta_{0} d\left(x_{n-1}, v\right)+L_{0} d\left(v, x_{n}\right)+\delta_{1} d\left(x_{n}, T v\right)+L_{1} d\left(T v, x_{n+1}\right) .
\end{aligned}
$$

Taking limits in the above inequality and using the fact that $d(v, T v)=0$, we deduce that $d\left(v, T^{2} v\right)$ is bounded above and below by zero, hence the assumption that $d\left(v, T^{2} v\right)>0$ cannot be true, it must be the case that $d\left(v, T^{2} v\right)=0$, that is, $T^{2} v=v$. It now follows that $v$ is also a fixed point of $T^{2}$. Now we show the fixed point is unique. Suppose $a=T a=T^{2} a$ and $b=T b=T^{2} b$, but $a \neq b$. From the second part of the theorem we deduce the following

$$
\begin{aligned}
d(a, b) & =d\left(T^{2} a, T^{2} b\right) \\
& \leq \delta_{0} d(a, b)+L_{0}^{*} d(a, T a)+\delta_{1} d(T a, T b)+L_{1}^{*} d\left(T a, T^{2} a\right)
\end{aligned}
$$




$$
\begin{aligned}
& =\delta_{0} d(a, b)+\delta_{1} d(a, b) \\
& \leq\left(\delta_{0}+\delta_{1}\right) d(a, b) .
\end{aligned}
$$

Since $1-\left(\delta_{0}+\delta_{1}\right) \neq 0$ and $d>0$, from the above inequality we must have $d(a, b)=0$ and hence $a=b$, which contradicts the assumption that $a \neq b$. Thus, the fixed point is unique.

\subsection{A best proximity point theorem}

Theorem 2.2. Let $(X, d)$ be a complete metric space, $G=(V(G), E(G))$ be a directed graph such that $V(G)=X$. Let $W$ and $V$ be nonempty closed subsets of $X$ with $W_{0}$ nonempty. Let $S: W \mapsto V$ be a non-self mapping satisfying the following properties:

(a) $S$ is proximally $G$-edge-preserving, continuous and G-proximal convex $(\delta, L)$ weak contraction such that $S\left(W_{0}\right) \subset V_{0}$.

(b) there exist $x_{0}, x_{1} \in W_{0}$ such that

$$
d\left(x_{1}, S x_{0}\right)=d(W, V), d\left(x_{2}, S x_{1}\right)=d\left(x_{2}, S^{2} x_{0}\right)=d(W, V) \text { and }\left(x_{0}, x_{1}\right) \in E(G) .
$$

Then $S$ has a best proximity point in $W$, that is, there exists an element $w \in W$ such that $d(w, S w)=d(W, V)$ and $d\left(w, S^{2} w\right)=d(W, V)$. Further the sequence $\left\{x_{n}\right\}$ defined by

$$
d\left(x_{n}, S x_{n-1}\right)=d(W, V) \text { and } d\left(x_{n+1}, S x_{n}\right)=d\left(x_{n+1}, S^{2} x_{n-1}\right)=d(W, V)
$$

for all $n \in \mathbb{N}$ converges to the element $w$.

Proof. From condition (b), there exist $x_{0}, x_{1} \in W_{0}$ such that

$$
d\left(x_{1}, S x_{0}\right)=d(W, V), d\left(x_{2}, S x_{1}\right)=d\left(x_{2}, S^{2} x_{0}\right)=d(W, V) \text { and }\left(x_{0}, x_{1}\right) \in E(G) .
$$

Since $S\left(W_{0}\right) \subseteq V_{0}$, we have $S x_{2} \in V_{0}$ and hence there exists $x_{3} \in W_{0}$ such that

$$
d\left(x_{3}, S x_{2}\right)=d(W, V) \text { and } d\left(x_{4}, S x_{3}\right)=d\left(x_{4}, S^{2} x_{2}\right)=d(W, V) .
$$

By the proximally $G$-edge preserving of $S$ and using both (1) and (2), we get

$$
\left(x_{3}, x_{4}\right),\left(x_{2}, x_{3}\right) \in E(G) .
$$


By continuing this process, we can form the sequence $\left\{x_{n}\right\}$ in $W_{0}$ such that

$$
\begin{array}{r}
d\left(x_{n}, S x_{n-1}\right)=d(W, V) \text { and } d\left(x_{n+1}, S x_{n}\right)=d\left(x_{n+1}, S^{2} x_{n-1}\right)=d(W, V) \\
\text { with }\left(x_{n-1}, x_{n}\right) \in E(G) \text {, for all } n \in \mathbb{N} .
\end{array}
$$

Next we show that $S$ has a best proximity point in $W$. Suppose there exists $n_{0} \in \mathbb{N}$ such that $x_{n_{0}}=x_{n_{0}+1}$. By using (3), we obtain that

$$
d\left(x_{n_{0}}, S x_{n_{0}}\right)=d\left(x_{n_{0}+1}, S x_{n_{0}}\right)=d(W, V)
$$

and

$$
d\left(x_{n_{0}}, S^{2} x_{n_{0}}\right)=d\left(x_{n_{0}+1}, S^{2} x_{n_{0}}\right)=d\left(x_{n_{0}+2}, S^{2} x_{n_{0}}\right)=d(W, V)
$$

and so $x_{n_{0}}$ is a best proximity point of $S$ and of $S^{2}$. Now we suppose that $x_{n-1} \neq x_{n}$ for all $n \in \mathbb{N}$. We show that $\left\{x_{n}\right\}$ is a Cauchy sequence in $W$. As $S$ is $G$-proximal convex $(\delta, L)$ weak contraction, and for each $n \in \mathbb{N}, \quad\left(x_{n-1}, x_{n}\right) \in E(G), d\left(x_{n}, S x_{n-1}\right)=$ $d(W, V)$ and $d\left(x_{n+1}, S x_{n}\right)=d\left(x_{n+1}, S^{2} x_{n-1}\right)=d(W, V)$, then we have

$$
\begin{aligned}
d\left(x_{n+1}, x_{n+2}\right) & \leq \delta_{0} d\left(x_{n-1}, x_{n}\right)+L_{0} d\left(x_{n}, x_{n}\right)+\delta_{1} d\left(x_{n}, x_{n+1}\right)+L_{1} d\left(x_{n+1}, x_{n+1}\right) \\
& =\delta_{0} d\left(x_{n-1}, x_{n}\right)+\delta_{1} d\left(x_{n}, x_{n+1}\right) \\
& \leq\left(\delta_{0}+\delta_{1}\right) \max \left\{d\left(x_{n-1}, x_{n}\right), d\left(x_{n}, x_{n+1}\right)\right\} \\
& =\left(\delta_{0}+\delta_{1}\right) d\left(x_{n}, x_{n+1}\right) .
\end{aligned}
$$

Now set $h:=\delta_{0}+\delta_{1}$. By the above inequality we have

$$
d\left(x_{1}, x_{2}\right) \leq h d\left(x_{0}, x_{1}\right)
$$

and hence

$$
d\left(x_{2}, x_{3}\right) \leq h^{2} d\left(x_{0}, x_{1}\right) .
$$

By induction, we deduce the following

$$
d\left(x_{n}, x_{n+1}\right) \leq h^{n} d\left(x_{0}, x_{1}\right)
$$

for all $n \in \mathbb{N} \cup\{0\}$. From (4), for each $m, n \in \mathbb{N}$ with $m>n$, we deduce the following 


$$
\begin{aligned}
d\left(x_{n}, x_{m}\right) & \leq d\left(x_{n}, x_{n+1}\right)+d\left(x_{n+1}, x_{n+2}\right)+\cdots+d\left(x_{m-1}, x_{m}\right) \\
& \leq h^{n} d\left(x_{0}, x_{1}\right)+h^{n+1} d\left(x_{0}, x_{1}\right)+\cdots+h^{m-1} d\left(x_{0}, x_{1}\right) \\
& =d\left(x_{0}, x_{1}\right) \sum_{i=n}^{m-1} h^{i} \\
& =\frac{h^{n}}{1-h} d\left(x_{0}, x_{1}\right) .
\end{aligned}
$$

Since $h \in(0,1)$, it follows that $\left\{x_{n}\right\}$ is a Cauchy sequence in $W$. Since $W$ is closed, there exists $w \in W$ such that $x_{n} \rightarrow w$. By continuity of $S$ and of $S^{2}$, we have $S x_{n} \rightarrow S w$ and $S^{2} x_{n} \rightarrow S^{2} w$ as $n \rightarrow \infty$. As the metric function is continuous, we obtain

$$
d\left(x_{n+1}, S x_{n}\right) \rightarrow d(w, S w) \text { as } n \rightarrow \infty
$$

and

$$
d\left(x_{n+2}, S x_{n+1}\right)=d\left(x_{n+2}, S^{2} x_{n}\right) \rightarrow d\left(w, S^{2} w\right) \text { as } n \rightarrow \infty .
$$

Similarly, by (3), we have

$$
d(w, S w)=d(W, V) \text { and } d\left(w, S^{2} w\right)=d(W, V) .
$$

It follows that $w \in W$ is a best proximity point of $S$ and of $S^{2}$. Moreover, the sequence $\left\{x_{n}\right\}$ defined by

$$
\begin{array}{r}
d\left(x_{n+1}, S x_{n}\right)=d(W, V) \text { and } d\left(x_{n+2}, S x_{n+1}\right)=d\left(x_{n+2}, S^{2} x_{n}\right)= \\
n(W, V), \\
n \in \mathbb{N} \cup\{0\}
\end{array}
$$

converges to an element $w$, and the proof is completed.

\section{Open Problem}

Definition 3.1. [10] Let $(X, d)$ be a metric space. A map $T: X \mapsto X$ is called a Reich contraction if there exist nonnegative constants $a, b, c$ with $a+b+c<1$ such that the following holds for all $x, y \in X$ 


$$
d(T x, T y) \leq a d(x, y)+b d(x, T x)+c d(y, T y) .
$$

Note if $T$ is a Reich contraction, then it also satisfies the following

$$
d(T x, T y) \leq(a+b+c) \max \{d(x, y), d(x, T x), d(y, T y)\} .
$$

Therefore we have the following

Definition 3.2. [11] Let $(X, d)$ be a metric space. A map $T: X \mapsto X$ is also a Reich contraction if there exists $k \in\left[0, \frac{1}{3}\right)$ such that the following holds for all $x, y \in X$

$$
d(T x, T y) \leq k[d(x, y)+d(x, T x)+d(y, T y)] .
$$

Related to the Reich contraction, the following was obtained

Theorem 3.3. [10] Let $(X, d)$ be a metric space, and $T: X \mapsto X$ be a Reich contraction. Then Thas a unique fixed point, provided $(X, d)$ is complete.

Now we introduced the following, as the Berinde characterization of the Reich contraction

Definition 3.4. [11] Let $(X, d)$ be a metric space. A map $T: X \mapsto X$ is called a Berinde weak Reich contraction, if there exists $\delta \in\left[0, \frac{1}{3}\right)$ and $L \geq 0$ such that the following holds for all $x, y \in X$

$$
d(T x, T y) \leq \delta[d(x, y)+d(x, T x)+d(y, T y)]+L d(y, T x) .
$$

Note that if $a=T a$ and $b=T b$, but $a \neq b$, then we have the following inequality from Definition 3.4

$$
\begin{aligned}
d(a, b) & =d(T a, T b) \\
& \leq \delta[d(a, b)+d(a, T a)+d(b, T b)]+L d(b, T a) \\
& =\delta[d(a, b)+d(a, a)+d(b, b)]+L d(b, a) \\
& =\delta d(a, b)+L d(b, a) \\
& =(\delta+L) d(a, b) .
\end{aligned}
$$


Unless $1-(\delta+L)>0$ we cannot conclude $a=b$. So we introduced the following as a way to "force" uniqueness of the fixed point.

Definition 3.5. [11] Let $(X, d)$ be a metric space. A map $T: X \mapsto X$ is called a $(\delta, 1-3 \delta)$ weak Reich contraction if the following holds for all $x, y \in X$ and $\delta \in\left(0, \frac{1}{3}\right)$

$$
d(T x, T y) \leq \delta[d(x, y)+d(x, T x)+d(y, T y)]+(1-3 \delta) d(y, T x) .
$$

Note that if $L=0$ in Definition 3.4, then we recover Definition 3.2. Note that for any $\delta \in\left(0, \frac{1}{3}\right)$, Definition 3.5 does not reduce to Definition 3.2.

The following result is previously known

Theorem 3.6. [11] Let $(X, d)$ be a metric space, and $T: X \mapsto X$ be a $(\delta, 1-3 \delta)$ weak Reich contraction. Then $T$ has a unique fixed point provided $X$ is complete.

Our first open problem introduces a so-called convex $(\delta, L)$ weak Reich contraction mapping theorem

Conjecture 3.7. Let $(X, d)$ be a metric space, and $T: X \mapsto X$ be a convex $(\delta, L)$ weak Reich contraction mapping, that is, T satisfies

$$
\begin{aligned}
d\left(T^{2} x, T^{2} y\right) \leq & \delta_{0}[d(x, y)+d(x, T x)+d(y, T y)]+L_{0} d(y, T x) \\
& +\delta_{1}\left[d(T x, T y)+d\left(T x, T^{2} x\right)+d\left(T y, T^{2} y\right)\right]+L_{1} d\left(T y, T^{2} x\right)
\end{aligned}
$$

for all $x, y \in X$ with $0<\delta_{0}, \delta_{1}<\frac{1}{3}, L_{0}, L_{1} \geq 0$, and $\delta_{0}+\delta_{1}<1$. If $(X, d)$ is complete, then the fixed point of $T$ exists. If in addition, $T$ is a convex $(\delta, L)$ weak Reich contraction such there exists $0<\delta_{0}, \delta_{1}<\frac{1}{3}, L_{0}^{*}, L_{1}^{*} \geq 0$, with $\delta_{0}+\delta_{1}<1$ satisfying

$$
\begin{aligned}
d\left(T^{2} x, T^{2} y\right) \leq & \delta_{0}[d(x, y)+d(x, T x)+d(y, T y)]+L_{0}^{*} d(x, T x) \\
& +\delta_{1}\left[d(T x, T y)+d\left(T x, T^{2} x\right)+d\left(T y, T^{2} y\right)\right]+L_{1}^{*} d\left(T x, T^{2} x\right),
\end{aligned}
$$

then the fixed point is unique. 
Sequel to the second open problem, we will need the following

Definition 3.8. Let $S: W \mapsto V$ be a non-self mapping. We say $S$ is a proximal convex $(\delta, L)$ weak Reich contraction if there exist $\delta_{0}, \delta_{1} \in\left(0, \frac{1}{3}\right), L_{0}, L_{1} \geq 0$, and $u_{1}, u_{2}, x, y \in W$ such that $d\left(u_{1}, S x\right)=d(W, V)$ and $d\left(u_{2}, S y\right)=d(W, V)$ implies

$$
\begin{aligned}
d\left(S u_{1}, S u_{2}\right) \leq & \delta_{0}\left[d(x, y)+d\left(x, u_{1}\right)+d\left(y, u_{2}\right)\right]+L_{0} d\left(y, u_{1}\right) \\
& +\delta_{1}\left[d(S x, S y)+d\left(S x, S u_{1}\right)+d\left(S y, S u_{2}\right)\right]+L_{1} d\left(S y, S u_{1}\right) .
\end{aligned}
$$

Definition 3.9. Let $(X, d)$ be a metric space, and $G=(V(G), E(G))$ be a directed graph such that $V(G)=X$. A non-self mapping $S: W \mapsto V$ is called a G-proximal convex $(\delta, L)$ weak Reich contraction, if there exists $\delta_{0}, \delta_{1} \in\left(0, \frac{1}{3}\right)$ and $L_{0}, L_{1} \geq 0$ such that $(x, y) \in E(G), d(u, S x)=d(W, V)$, and $d(v, S y)=d(W, V)$ implies

$$
\begin{aligned}
d(S u, S v) \leq & \delta_{0}[d(x, y)+d(x, u)+d(y, v)]+L_{0} d(y, u) \\
& +\delta_{1}[d(S x, S y)+d(S x, S u)+d(S y, S v)]+L_{1} d(S y, S u),
\end{aligned}
$$

where $x, y, u, v \in W$.

Now we have the following which can be regarded as the non-self counterpart to Conjecture 3.7 in graphic language

Conjecture 3.10. Let $(X, d)$ be a complete metric space, $G=(V(G), E(G))$ be a directed graph such that $V(G)=X$. Let $W$ and $V$ be nonempty closed subsets of $X$ with $W_{0}$ nonempty. Let $S: W \mapsto V$ be a non-self mapping satisfying the following properties:

(a) $S$ is proximally $G$-edge-preserving, continuous and G-proximal convex $(\delta, L)$ weak Reich contraction such that $S\left(W_{0}\right) \subset V_{0}$

(b) there exist $x_{0}, x_{1} \in W_{0}$ such that

$$
d\left(x_{1}, S x_{0}\right)=d(W, V), d\left(x_{2}, S x_{1}\right)=d\left(x_{2}, S^{2} x_{0}\right)=d(W, V) \text { and }\left(x_{0}, x_{1}\right) \in E(G) .
$$

Then $S$ has a best proximity point in $W$, that is, there exists an element $w \in W$ such that $d(w, S w)=d(W, V)$ and $d\left(w, S^{2} w\right)=d(W, V)$. Further the sequence $\left\{x_{n}\right\}$ defined 
by

$$
d\left(x_{n}, S x_{n-1}\right)=d(W, V) \text { and } d\left(x_{n+1}, S x_{n}\right)=d\left(x_{n+1}, S^{2} x_{n-1}\right)=d(W, V)
$$

for all $n \in \mathbb{N}$ converges to the element $w$.

\section{References}

[1] V. Berinde, Approximation fixed points of weak contractions using the Picard iteration, Nonlinear Anal. Forum 9(1) (2004), 43-53.

[2] Vasile I. Istratescu, Some fixed point theorems for convex contraction mappings and convex nonexpansive mappings (I), Libertas Math. 1 (1981), 151-163.

[3] Clement Boateng Ampadu, A new proof of the convex contraction mapping theorem in metric spaces, Internat. J. Math. Arch., to appear.

https://drive.google.com/file/d/0BwtkpMtWoUlEV0d4QUhnaVlqOHc/view

[4] Clement Ampadu, Fixed Point Theory for Higher-Order Mappings, lulu.com, 2016. ISBN: 5800118959925

[5] Jeffery Ezearn, Higher-order Lipschitz mappings, Fixed Point Theory Appl. 2015, 2015:88, 18 pp.

[6] Vasile Berinde, On the approximation of fixed points of weak contractive mappings, Carpathian J. Math. 19(1) (2003), 7-22.

[7] K. Fan, Extensions of two fixed point theorems of F.E. Browder, Math. Z. 112 (1969) 234-240.

[8] Chalongchai Klanarong and Suthep Suantai, Best proximity point theorems for $G$-proximal generalized contraction in complete metric spaces endowed with graphs, Thai J. Math. 15(1) (2017), 261-276.

[9] S. S. Basha, Best proximity points: optimal solutions, J. Optim. Theory Appl. 151(1) (2011), 210-216.

[10] S. Reich, Some remarks concerning contraction mappings, Canad. Math. Bull. 14 (1971), 121-124.

[11] Clement Boateng Ampadu, An almost Berinde Reich mapping theorem with unique fixed point, Global J. Pure Appl. Math., to appear.

https://drive.google.com/file/d/1TecJ2bVkpKRsCtCZZ8fhlZ8h04yptsFU/view 